\title{
Oral Session 10: Countermeasures
}

\section{0-1 [\#3038]}

\section{Validation of centrifugation as a countermeasure for otolith deconditioning during spaceflight: Preliminary data of the ESA SPIN study}

K.I. Buytaert ${ }^{\mathrm{a}}$, H.G. MacDougall ${ }^{\mathrm{b}}$, P.-F. Migeotte ${ }^{\mathrm{c}}$, N. Pattyn ${ }^{c}$, G. Clement ${ }^{\mathrm{d}}$, S.T. Moore ${ }^{\mathrm{e}}$, P.H. Van de Heyning $^{\mathrm{a}}$ and F.L. Wuyts ${ }^{\mathrm{a}}$

a Antwerp University Research centre for Equilibrium and Aerospace (AUREA), ENT Department, Antwerp University Hospital, University of Antwerp, Wilrijkstraat 10, 2650, Edegem, Belgium. E-mail: kristof. buytaert@ua.ac.be

${ }^{\mathrm{b}}$ Department of Psychology, University of Sydney, Sydney, NSW, Australia

c Signal and Image Centre, Electrical Engineering Department, Royal Military Academy, Brussels, Belgium ${ }^{\mathrm{d}}$ Faculty of Medicine, University of Toulouse, Toulouse, France

${ }^{\mathrm{e}}$ Mount Sinai School of Medicine, New York, USA

In the framework of further space exploration, countermeasures to combat the drawbacks of human space flights are essential. The here described study focuses on the influence of microgravity on the otolith-ocular reflex and aims to verify the hypothesis of artificial gravity being an adequate countermeasure for the deconditioning of the aforementioned reflex. The socalled SPIN-study serves as control experiment for the Neurolab mission (STS-90) during which 4 crewmembers of the space shuttle were subjected to in-flight centrifugation on the visual and vestibular investigation system (VVIS). After their mission, they did not suffer from orthostatic intolerance and spatial disorientation. In addition, the relevant parameters of the otolith-ocular interaction remained unaffected. For this study cosmonauts from a long duration stay in the International Space Station that were not centrifuged in-flight were tested on the VVIS ( $1 \mathrm{~g}$ centripetal interaural acceleration; consecutive right-ear-out anti-clockwise and leftear-out clockwise measurement) on 6 different days. Three measurements were scheduled about one month and a half prior to launch and the remaining 3 immediately after their return from space (on $\mathrm{R}+1, \mathrm{R}+4, \mathrm{R}+9$; $\mathrm{R}=$ return day from space). The ocular counter roll was measured on several steady state moments before, during and after the rotation using infrared video goggles. The perception of verticality was monitored using an ultrasound system. Optokinetic stimuli were offered during the rotation and the change of the orientation of the velocity vector of the optokinetic nystagmus was investigated. Analysis of the results indicates a large inter subject variability. The preliminary results of 4 cosmonauts will be shown.

\section{0-2 [\#3053]}

Galvanic vestibular stimulation replicates post-flight deficits in posture, gait, gaze and operator proficiency

S.T. Moore ${ }^{\mathrm{a}}$, H.G. MacDougall ${ }^{\mathrm{b}}$, V. Dilda ${ }^{\mathrm{a}}$, I.S. Curthoys $^{\mathrm{b}}$, J. Bloomberg ${ }^{\mathrm{c}}$, H.S. Cohen ${ }^{\mathrm{d}}$ and F.O. Black $^{\mathrm{e}}$

${ }^{\mathrm{a}}$ Human Aerospace Laboratory, Mount Sinai School of Medicine, Department of Neurology, New York NY, USA.E-mail: steven.moore@mssm.edu

${ }^{\mathrm{b}}$ School of Psychology, University of Sydney, Australia ${ }^{\mathrm{c}}$ Neuroscience Laboratory, NASA Johnson Space Center, Houston, TX, USA

${ }^{\mathrm{d}}$ Department of Otolaryngology, Baylor College of Medicine, Houston, TX, USA

${ }^{\mathrm{e}}$ Neurotology Research, Legacy Health System, Portland, OR, USA 
Background: Crewmembers returning to a gravitational environment after prolonged microgravity exposure exhibit decrements in balance, locomotion, gaze and manual control. Currently there is no ground ana$\log$ for the sensorimotor effects of spaceflight. Here we summarize 5 years of development and validation of a novel analog of post-flight sensorimotor dysfunction utilizing Galvanic vestibular stimulation (GVS).

Methods: The GVS system delivers a pseudorandom (sum of sines, $0.16,0.32,0.43,0.61 \mathrm{~Hz}, \max \pm$ $5 \mathrm{~mA}$ ) transmastoidal current via surface electrodes [1, 2].

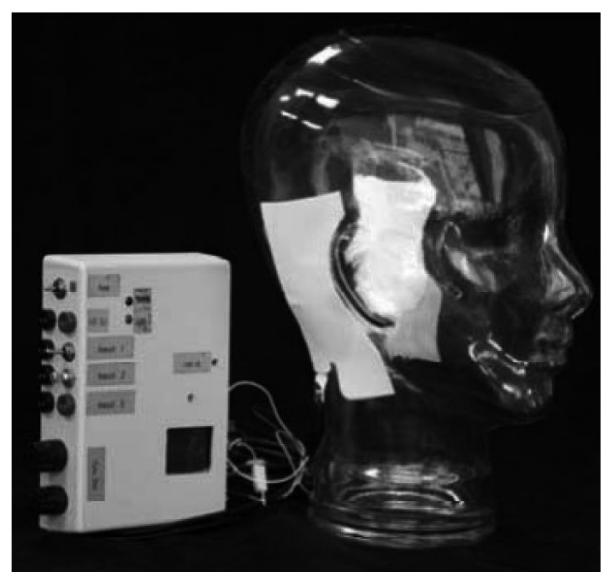

Fig. 1. The GVS system. (Colours are visible in the online version of the article; http://dx.doi.org/10.3233/VES-2010-0411)

\section{Results:}

Posture

The equilibrium scores in normal subjects $(N=12)$ exposed to acute GVS during computerized dynamic posturography (CDP) accurately reproduced deficits in anterioposterior sway observed in returning shuttle crewmembers [1].

Gait:

Head stabilization (linear vestibulo-collic reflex) was degraded in subjects $(N=20)$ exposed to GVS during treadmill locomotion, equivalent to that observed in shuttle astronauts on landing day. Time to complete an obstacle course increased $21 \%$ during GVS, equivalent to an ISS astronaut 5 days post-landing [2].

Dynamic Visual Acuity (DVA):

DVA decreased by $0.06 \log$ MAR with GVS, equivalent to the reduction in acuity observed in astronauts 6 days after return from extended missions aboard the ISS [2].

Operator Proficiency:

Pilot performance was assessed during shuttle landings in the Vertical Motion Simulator (NASA Ames).
Subjects $(N=11)$ flew 8 pairs of identical landing profiles with and without GVS. Touchdown speed wason target (204 kts) without GVS but increased significantly during GVS exposure and was at the upper limit (209 kts) of the target range. The rate of unsuccessful (crash) landings tripled, and hard landings ( $>214$ kts) almost doubled, with GVS relative to the no-GVS baseline [3]. These results were consistent with a review of the first 100 shuttle missions [4], which reported touchdown speed above specified limits in $20 \%$ of landings.

Conclusion: GVS is an effective analog of sensorimotor deficits following spaceflight.

Acknowledgement: Supported by NASA grant NNJ 04HF51G, and NSBRI grants SM00801 and SA01603 through NASA NCC 9-58.

\section{References}

[1] MacDougall et al. (2006) Exp Brain Res 172: 208-220.

[2] Moore et al. (2006) Exp Brain Res 174: 647-659.

[3] Moore et al. (2010) Aviat Space Environ Med In Press $(11 / 26 / 10)$.

[4] Moore et al. (2008) Aviat Space Environ Med 79: 888-898.

\section{0-3 [\#3013]}

\section{Stimulus characteristics for vestibular stochastic resonance to improve balance function}

A.P. Mulavara ${ }^{\mathrm{a}}$, M.J. Fiedler ${ }^{\mathrm{b}}$, I. Kofman ${ }^{\mathrm{b}}$, B. Peters ${ }^{\mathrm{b}}$, S.J. Wood ${ }^{\text {a }}$, J.M. Serrador ${ }^{\mathrm{c}}$, H. Cohen ${ }^{\mathrm{d}}$, M.F. Reschke ${ }^{\mathrm{e}}$ and J.J. Bloomberg ${ }^{\mathrm{e}}$

${ }^{a}$ Universities Space Research Association, Houston, $T X, U S A$

${ }^{\mathrm{b}}$ Wyle Integrated Science and Engineering Group, Houston, TX, USA

${ }^{\mathrm{c}}$ Department of Veterans Affairs NJ Healthcare System, East Orange, NJ, USA

${ }^{\mathrm{d}}$ Baylor College of Medicine, Houston, TX, USA

${ }^{\mathrm{e}}$ NASA Johnson Space Center, Houston, TX, USA

Stochastic resonance (SR) is a mechanism by which noise can assist and enhance the response of neural systems to relevant sensory signals. Studies have shown that imperceptible stochastic vestibular electrical stimulation, when applied to normal young and elderly subjects, significantly improved their ocular stabilization reflexes in response to whole-body tilt as well as balance performance during postural disturbances. The 
goal of this study was to optimize the amplitude characteristics of the stochastic vestibular signals for balance performance during standing on an unstable surface. Subjects performed a standard balance task of standing on a block of foam with their eyes closed. Bipolar stochastic electrical stimulation was applied to the vestibular system using a constant current stimulator through electrodes placed over the mastoid process behind the ears. Amplitude of the signals varied in the range of 0-700 microamperes. Balance performance was measured using a force plate under the foam block, and inertial motion sensors placed on the trunk and head. Balance performance with stimulation was significantly improved in the range of 10\%-25\% compared with no stimulation. The signal amplitude at which performance was maximized was in the range of 100-400 microamperes. Optimization of the amplitude of the stochastic signals for maximizing balance performance will have a significant impact on development of vestibular SR as a unique system to aid recovery of function in astronauts after long-duration space flight or in patients with balance disorders.

Acknowledgement: This study was supported in part by a grant from the NSBRI through NASA NCC 9-58 (SA02001). 